\title{
Argumentación y conocimiento científico escolar ${ }^{1}$
}

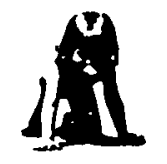 \\ Resumen
}

$\mathrm{M}^{2}$ ANTONIA CANDELA

Centro de Investigación y de Estudios Avanzados

En este articulo se analiza el contexto interactivo que propicia la participación de los alumnos en la construcción social del conocimiento de ciencias naturales en la escuela primaria. La descripción se centra en las intervenciones de los alumnos, elaboradas en interacción con el docente, en las que expresan explicaciones alternativas y argumentan sus ideas.

Palabras claves: Argumentación, Explicaciones, Analogías, Construcción social del conocimiento, Ciencia, Etnografia, Primaria, México, Interacción maestro-alumnos.

\section{Argumentation and knowledge in science classes}

\section{Abstract}

This paper analyses the interactive context that contributes to creating the conditions for student participation in the social construction of science knowledge in primary school. The description centers on the children's argumentations and alternative explanations in interaction with the teacher.

Keywords: Argumentation, Explanations, Analogies, Social construction of knowledge, Science, Ethnography, Elementary education, Mexico, Teacher-student interaction.

Agradecimientos: Este artículo presenta parte de los resultados de un proyecto que recibió financiamiento del Consejo Nacional de Ciencia y Tecnología de México con el Convenio PCEDCNA-0502/2. Agradezco los comentarios de mis colegas Ruth Mercado, Ruth Paradise, Rafael Quiroz y Elsie Rockwell, así como las observaciones de Derek Edwards, que han sido de gran utilidad para la orientación de mi trabajo.

Dirección de la autora: Departamento de Investigaciones Educativas. Centro de Investigación y de Estudios Avanzados del Instituto Politécnico Nacional. Apartado Postal 19-197. México, D. F. 03900.

Original recibido: Marzo 1991. Revisión recibida: Mayo 1991. Aceptado: Mayo 1991 


\section{4}

INTRODUCCION

En el transcurso de las últimas décadas fueron desarrolladas gran cantidad de propuestas didácticas para la enseñanza de la ciencia sustentadas en estudios experimentales acerca de las concepciones del mundo físico que tienen los niños (Piaget, 1973; Viennot, 1979; Driver, Guesne y Thibergien, 1985; Driver, 1986) ${ }^{2}$. Estas iniciativas no lograron sin embargo, su propósito de incidir en la práctica escolar, de la manera como se lo proponía. Esto puede deberse, entre otros factores, al desconocimiento de las condiciones concretas en las que iban a operar. El estudio de dichas condiciones es importante en tanto el aprendizaje significativo depende no sólo del desarrollo cognitivo de los sujetos y de sus ideas previas en torno a los contenidos, sino también del contexto social interactivo en el que se produce (Cicourel, 1974; Bruner, 1984; Coll, 1986).

Si bien se han destinado grandes recursos económicos y humanos para desarrollar modelos que mejoren la enseñanza de las ciencias, son pocos los trabajos (Rockwel y Gálvez, 1982; Candela, 1989, 1990; Edwards, 1990; Hernández, 1989) sobre la construcción del conocimiento de ciencias naturales en el contexto escolar cotidiano. El análisis de lo que se enseña, cómo se enseña y las condiciones que se generan en la enseñanza habitual para el aprendizaje de las ciencias, es necesario para la elaboración de propuestas que pretendan mejorar la práctica docente.

Para Barnes se aprende, no sólo al escuchar, sino al discutir activamente, al expresar opiniones, defender puntos de vista en el debate, etc. Por lo tanto, estudiando la interacción profesor-alumno en el aula se pueden analizar las situaciones que abren o cierran diferentes posibilidades de aprendizaje para los alumnos (Barnes, 1971). En este trabajo se analizan estas situaciones pero no se aborda su efecto en los procesos cognitivos individuales.

El estudio de los procesos educativos en el contexto escolar exige la utilización de recursos metodológicos adecuados. Como dice Edwards (en prensa), el uso de la entrevista clínica permite aproximarse a la dimensión individual del pensamiento, a las estructuras cognitivas del individuo, pero hace difícil inferir el componente social del pensamiento, la organización local, situacional y pragmática del razonamiento conceptual. En cambio, el análisis del habla en la dinámica de la interacción social revela la dimensión social y contextualizada del pensamiento de los niños, aunque no da información suficiente para hacer inferencias sobre el aprendizaje o acerca de las representaciones cognitivas individuales.

Por construcción social del conocimiento en este trabajo no se entiende (aunque comparto la concepción vygotskyana) la construcción individual del conocimiento a partir del lenguaje y de las interacciones sociales, sino la elaboración del conocimiento que se expresa en las interacciones orales y que es compartido y construido colectivamente en el aula escolar (Edwards y Mercer, 1988).

Se asume que, aunque el docente mantenga el control del conocimiento en el salón de clases, los alumnos son sujetos activos capaces de contribuir con sus intervenciones a la construcción del conocimiento compartido. Con base en lo anterior, se intenta analizar tanto la participación de los alumnos 
en la construcción del conocimiento compartido, como el efecto de sus contribuciones en la dinámica de la interacción.

El texto analiza la dinámica de la interacción entre el maestro y los alumnos, en una situación cotidiana de enseñanza de la ciencia, en la que aparecen formulaciones orales de los alumnos que pueden contribuir a su formación científica ${ }^{3}$. En particular se estudian aquellas situaciones en las que los niños formulan explicaciones diversas y argumentan sus puntos de vista. También se analizan algunas condiciones (intervenciones didácticas del docente, relevancia del contenido, relación entre pares, intencionalidad) que propician las situaciones mencionadas.

La formulación de explicaciones alternativas y la argumentación de las ideas son centrales para la formación científica. Einstein llamó a los científicos personas con pasión por explicar. Para la nueva sociología del conocimiento científico (Gilbert y Mulkay, 1984; Longino, 1990) el análisis de la argumentación es cada vez más importante. Esto es así ya que el conocimiento científico es entendido como un sistema socialmente construido de comprensiones, suposiciones y procedimientos compartidos por una comunidad (Phillips, 1984) y no como el producto de la aplicación de un "método científico" impersonal, invulnerable y «objetivo" que siempre conduce al conocimiento "verdaderom. Por otro lado, la organización argumentativa del discurso está en la base de una parte importante del razonamiento conceptual (Billig, 1987).

Por argumentación se entiende la articulación de intervenciones, dentro de un discurso, con la intención de convencer a otros sobre un punto de vista (Billig, 1987). Implica, por tanto, que existen diferentes opiniones sobre algún tópico. Por eso, argumentar es presentar una postura con la conciencia de que existe una opinión, implícita o explícita, diferente a la propia (Leith y Myerson, 1989).

Las intervenciones argumentativas ponen en juego conocimientos previos y los relacionan en formas variadas, modificando variables y situaciones para articular razones que convenzan. El razonamiento lógico puede ser visto como una argumentación con uno mismo. En una argumentación las intervenciones están enlazadas por medio de la confrontación y, por tanto, lo que alguien dice se vincula, en un debate, con lo que otro expresa (Edwards, 1990a).

El conocimiento socialmente construido en el aula se puede ver, en ciertos momentos, como un encuentro de argumentaciones explícitas e implícitas que tienen que ser negociadas para mantener la comunicación. Las expresiones orales se toman entonces como significados construidos en un contexto de interacción, como productos de una confrontación en relación con otros, con una posición precisa en la conversación y no como evidencia de modelos cognitivos personales.

\section{ENFOQUE METODOLOGICO}

Para aproximarnos al estudio del conocimiento de ciencias construido y compartido en el salón de clases adoptamos una perspectiva etnográfica que permite hacer una reconstrucción de carácter cualitativo y descriptivo de lo que se dice y hace en un aula. 
Los datos empíricos utilizados en la investigación fueron obtenidos de registros etnográficos realizados en los salones de clase de una escuela primaria pública ubicada en una zona marginal de la periferia de la Ciudad de México ${ }^{4}$. Visitamos esta institución dos veces por semana durante un poco más de seis meses e hicimos observaciones del trabajo de 13 maestros. Los registros fueron realizados a partir de notas de las clases de ciencias naturales elaboradas por el investigador. Las secuencias de más interés para el análisis se completaron con transcripciones de la sesión grabada.

El registro de la clase completa, que se analiza, permite seguir la secuencia de pautas interactivas que ayudan a encontrar el significado de lo dicho en función del contexto en el que el lenguaje es usado. La unidad de análisis son las secuencias, algunas veces resumidas y otras textuales, en las que se van describiendo y analizando tanto las características de los significados construidos como la dinámica de la interacción.

\section{ANALISIS DE LA CïASE}

El Sistema Solar es un tema escolar que se trabaja desde $2 .^{\circ}$ grado de primaria, por lo que estos alumnos de $50^{\circ}$ grado (alrededor de 40 , de 10 a 11 años de edad y nivel socioeconómico bajo), ya lo habían abordado varias veces en la escuela. Es probable que el maestro haya escogido este tema con el propósito de presentar una especie de «clase modelo» el día en que fue observado. Sin embargo, hay que aclarar que este profesor no tuvo ningún tipo de entrenamiento especial ni se le hicieron sugerencias para dar la clase. Sólo se le pidió que diera su clase como siempre lo hacía.

El profesor, de unos 24 años, con cuatro años de experiencia docente, es considerado en la escuela como un buen maestro «tradicional», porque mantiene al grupo disciplinado y lleva un estrech control del proceso de interacción

En fecha anterior a la sesión registrada el maestro pidió a los niños que leyeran la lección correspondiente a El Sistema Solar en su Libro de Texto (que en México es el texto oficial y obligatorio para todas las escuelas primarias del país) de Ciencias Naturales de $50^{\circ}$ grado.

La observación que describimos se realizó en el penúltimo mes del año escolar y se puede apreciar una estructura organizativa integrada donde se comparten las reglas de la interacción.

\section{Secuencia 1: Estableciendo las pautas del diálogo}

M. "Vamos a empezar. La clase de hoy es...»

Ao. «Sistema Solar»

M. «Habíamos dejado que leyeran de tarea de la página 78 a la 86 . Aparte de que leyeran les dije que me trajeran las dudas que tuvieran... A ver, dudas." (silencio)

M. « Nadie tiene dudas?" (nadie contesta)

M. "Leti"

Leti. "Cuando la niña dice que estaba viendo el cielo, ¿lo que soñó es verdad o era su pura imaginación?»

M. «Bueno, aqui vamos a empezar con lo siguiente: cuando Juanita y $\mathrm{Ca}$ nica nos hablan que hay una estrella y..."

As. «Planetas» (a coro) 
M. «En todos los años les han hablado del...»

As. «Sistema Solar»

M. "Ustedes han ido al planetario. ¿Qué hay alrededor de las estrellas?"

As. «Meteoritos», «Asteroides», «Cometas»

En esta primera secuencia de la clase se observa que los alumnos están habituados a seguir dinámicas de intervención, como completar las frases del maestro.

Cuando el maestro hace una pregunta abierta sobre las dudas, los niños no responden, como si esperaran más pistas para saber cómo intervenir.

Sin responder a la aclaración que pide Leti ${ }^{5}$, el maestro retoma la dinámica de frases inconclusas para reubicar así los términos en los que debe darse la interacción. Se trata de que los alumnos mencionen los componentes del Sistema Solar. La pregunta sobre las dudas puede ser leída como el anuncio de lo que, fuera de clase, el maestro mencionó como su intención: "que se entienda bien todo lo que se da», «no dejar lagunas», «tratar de resolver las dudas de los niños». Por lo tanto, parece quedar planteado que ésta es una clase de repaso donde también se trata de aclarar dudas. Los niños continúan mencionando lo que, según ellos, hay alrededor de las estrellas.

\section{Secuencia 2: Conocimiento escolar y extraescolar}

Aa. "Yo vi, en una caricatura, que también hay remolinos que se iban a comer a la Tierra”

Ao. "Hoyos negros"

M. "QQué es un hoyo negro?"

Ao. «Es un pedazo donde está una estrella que se apagó»

M. «Como un remolino dice (nombra a la alumna) o como un pedazo donde está una estrella que se apagó (menciona el nombre del alumno)»

En esta secuencia llama la atención la iniciativa de una alumna de incluir en la interacción conocimiento extraescolar que no está en el Libro de Texto y que, probablemente, vio en la TV. Este conocimiento parece ser compartido por otros alumnos, pues un niño aclara que la descripción corresponde a los hoyos negros y otro más explica lo que es un hoyo negro.

El docente no sólo permite que los niños expresen conocimientos extraescolares sino que los incorpora al discurso escolar (pide que expliquen lo que entienden por hoyo negro). Es posible que aceptar la información que los niños manejan en su entorno social ayude a que ellos adquieran confianza en su conocimiento y que lo expresen en clase.

El maestro no ratifica ni niega la validez de lo que los niños dicen, como es frecuente que suceda en las escuelas, sino que retoma las dos descripciones y las confronta. Aceptar que haya opiniones distintas y compararlas con el fin de identificar las diferencias entre ellas es necesario para que haya debate y, por tanto, argumentación. Sin embargo, el maestro no da tiempo para expresar las ideas que pudieran surgir de la confrontación, sino que hace una pregunta para retomar la enumeración de lo que hay en el Sistema Solar. Abre así una posibilidad de debate, pero la cierra de inmediato.

En una especie de repaso, el maestro hace preguntas que gran parte de los niños responde, muchas de ellas a coro. Pregunta dónde está el Sistema Solar, qué astros hay en él, cuáles son los planetas que tienen satélites y 
cuáles los que no, pide que describan los movimientos de rotación y traslación, que digan la forma de las órbitas planetarias y que mencionen el nombre y el orden de los planetas con respecto al Sol.

En este repaso los alumnos participan aparentemente de manera mecánica y ritualizada sobre un conocimiento escolar memorizado acerca del Sistema Solar. Siguen con tanta fluidez la dinámica de la interacción que, al leer el registro, otros maestros comentan: "parece que ya lo sabían de memoria», «todo está como muy bien planeado, bien repasado", "es raro que todos los niños estén interesados».

Continúa el intercambio de preguntas y respuestas sobre el orden de los planetas con respecto al Sol.

\section{Secuencia 3: Analogia como recurso argumentativo}

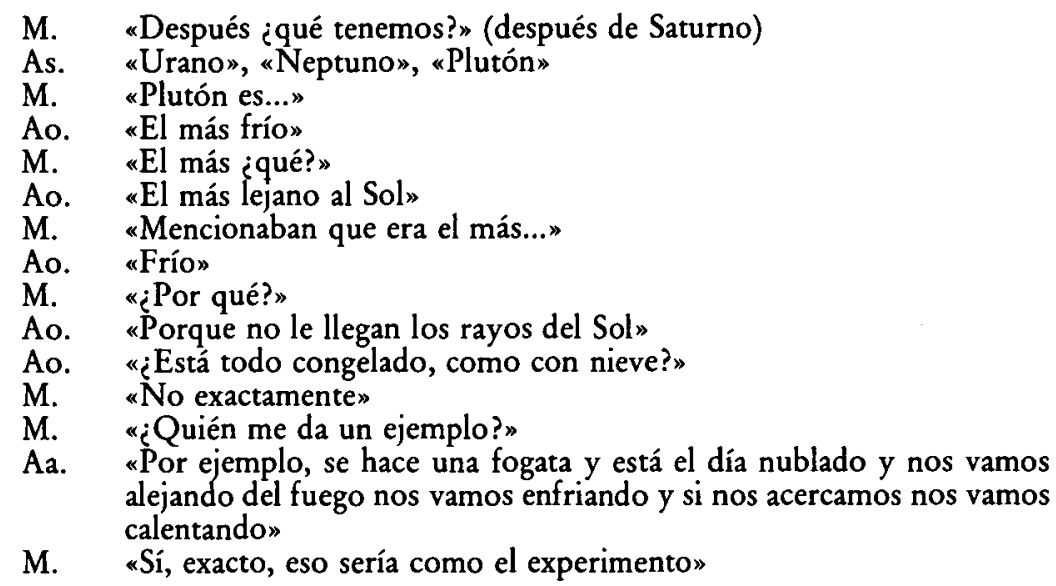

El maestro conduce la secuencia de las intervenciones de los alumnos, repite las preguntas para indicar que quiere otra respuesta e incluso deja frases inconclusas para buscar respuestas definidas. Con esta estructura cerrada conduce a los niños para que establezcan o expresen relaciones entre variables (temperatura y distancia a la fuente calorífica) y para que argumenten sus afirmaciones.

Al inicio de esta interacción los niños aprecian dos características de Plutón (el más lejano al Sol y el más frío de los planetas). Después de que el maestro pregunta "¿Por qué?», un alumno contesta: «Porque no le llegan los rayos del Sol». Esta es una explicación que utiliza relaciones causales y que, al mismo tiempo, opera como un argumento para justificar el vínculo entre distancia y temperatura. El argumento conlleva conceptos implícitos como que los «rayos" del Sol calientan a los planetas.

En el Libro de Texto se propone como experimento poner un foco encendido y colocar bolitas de plastilina en las posiciones relativas de los planetas; después se pregunta cuáles son las bolitas que están más calientes y cuáles más frías y qué diferencia habrá entre la temperatura de los planetas que están más cerca y los que están más lejos del Sol. La actividad, aunque sólo haya sido leída, puede haber sugerido la relación entre distancia al Sol y temperatura relativa de los planetas.

La pregunta de un alumno de si en Plutón «está todo congelado como 
con nieve» parece mostrar la necesidad de construir representaciones con base en analogías conocidas que les permitan hacer inferencias e imaginar lo que quieren decir las palabras (en este caso, "el más frío").

La pregunta del maestro «¿Quién me da un ejemplo?» promueve que los alumnos busquen analogías para validar sus afirmaciones. Para un observador externo resulta difícil comprender cómo entienden los niños lo que el maestro quiere que hagan a partir de esta pregunta. Sin embargo, la respuesta de la niña muestra que existen comprensiones compartidas sobre la forma de participación. El docente confirma que la respuesta es la que él esperaba.

El ejemplo de la niña para explicar la correlación entre la temperatura y la distancia a la fuente de calor opera como una forma de argumentar la validez de esta relación a través de la analogía con una experiencia cotidiana. La niña no utiliza el ejemplo del Libro sino una situación vivencial que probablemente le resulta más convincente.

Podríamos decir que esta interacción social propicia que se manifiesten aspectos fundamentales para la formación científica: a) elaboración de explicaciones causales que operan como argumento («Porque no le llegan los rayos del Sol") para justificar la relación entre variables (temperatura y distancia) y b) la argumentación sobre la explicación propuesta utilizando como prueba la analogía con una experiencia cotidiana.

En este caso (semejante a los que muestra Edwards, 1990b), el control del maestro sobre la dinámica de las intervenciones en el aula va estableciendo la estructura del conocimiento. Sin embargo, mientras que en los ejemplos de Edwards las intervenciones del docente contribuyen a fragmentar las contribuciones de los alumnos en segmentos parcializados acumulativos, en éste el maestro propicia una construcción argumentativa del conocimiento al pedir que los niños vinculen variables por medio de relaciones de causa-efecto y busquen pruebas de sus afirmaciones por analogía. Por tanto, con una estructura cerrada en la forma de la interacción, el maestro propicia una elaboración reflexiva y abierta del conocimiento.

El maestro toma la iniciativa para continuar la clase con una representación del Sistema Solar que es común observar cuando se enseña este tema en las escuelas primarias de México.

\section{Secuencia 5: El referente físico}

M. «A ver, quiero que me hagan el funcionamiento del Sistema Solar»

Van saliendo niños al frente del salón, unos voluntariamente y otros elegidos por el maestro.

M. "Juan Gabriel va a ser el Sol» Los niños se ríen probablemente porque Juan Gabriel es gordito.

M. "¿Cómo identificaremos a Guillermo que es Saturno?»

Ao. "Con un aro"

M. "Van viendo quién tendría más calor y quién más frío»

Los niños hacen una representación del movimiento de rotación y de traslación alrededor del Sol, pero se tienen que mover en un espacio muy reducido del aula.

Ao. «Todos andamos por diferente lugar porque si no chocaríamos»

Ao. "¿Por qué no chocan?" 
M. «Qué guardan entre unos y otros?»

Ao. "Distancia"

M. "Si se salen de su órbita sí llegarían a poder chocar»

Es probable que la falta de espacio en el salón de clases influya para que surja el comentario de un alumno sobre la posibilidad de chocar entre ellos así como la duda de otro niño que pregunta por qué no chocan los planetas.

Esta es la primera interrogante significativa que surge en el grupo después de que los alumnos han mencionado los movimientos y las posiciones de los planetas. En este caso la repetición de información memorizada no bloquea la posibilidad de que aparezcan preguntas sobre las causas del fenómeno. Los niños ya saben cómo se mueven los planetas en el Sistema Solar; ahora quieren saber por qué se mueven así. Es probable que el contexto interactivo, que desde el principio es planteado por el maestro como un repaso en donde se trata de resolver las dudas de los niños, contribuya a propiciar la aparición de estas interrogantes.

A la pregunta de por qué no chocan los planetas el maestro responde con otra pregunta que lleva implícita una respuesta que no explica, sino que conduce a una descripción con rasgos de finalismo. No chocan porque están en órbitas que guardan distancia ${ }^{6}$. Continúa la clase:

\section{Secuencia 6: Contraejemplos}

M. «¿Por qué no giran así?»

El maestro mueve a un niño en la órbita pero haciendo que dé siempre la cara al Sol.

Aa. "Porque sólo le daría calor de un lado y del otro frío"

Ao. "Porque sólo habría estaciones de un lado"

Ao. "Porque no habría día y noche"

M. "Muy bien, pero ... a ver»

Ahora el maestro hace que el niño rote y se traslade con diferentes velocidades, como lo hacen los planetas.

Ao. "Porque hay los dos movimientos"

Ao. "¿Qué pasaría si los planetas se pararan?»

M. «Se irían por el espacio» (dice después de un silencio)

El maestro utiliza un contraejemplo para tratar de convencer, por reducción al absurdo, sobre la validez del modelo de movimiento de los planetas. Aunque sigue buscando que los niños respondan lo que él espera, el sentido de su intervención es argumentativo porque el contraejemplo opera como un punto de vista distinto que tiene que ser cuestionado con razones. El contraejemplo consiste en la modificación de la velocidad de rotación de un planeta y obliga a que los niños analicen la relación entre ésta variable y los efectos que produciría su alteración. Los alumnos argumentan analizando que si la rotación tuviera el mismo período que la translación no habría día y noche, un lado estaría caliente y otro frío y sólo habría estaciones de un lado, cosa que no ocurre en la Tierra. Estos efectos no corresponden a la realidad, son absurdos. En esto consiste la argumentación por reducción al absurdo. 
Con la pregunta de qué pasaría si los planetas se pararan, un alumno imita la forma de presentación del conocimiento que hace el maestro. Trata de entender la dinámica del sistema alterando variables y analizando situaciones de excepción. El maestro se queda en silencio, como pensando, y responde «Se irían por el espacio", dando la impresión de que en ese momento construye la respuesta. La clase continúa:

\section{Secuencia 7: Confrontación de puntos de vista}

Aa1. "¿Por qué no nos caemos?"

M. "A ver, ¿quién sabe eso?"

Ao. "Porque hay una fuerza magnética que nos sostiene a nosotros."

Aa1. «Pero ¿quién sostiene a la Tierra?»

Aa. "Porque al girar genera aire que la sostiene."

La niña repite su explicación al frente del salón, por indicación del maestro.

Ao. «Hay una fuerza magnética que nos jala para abajo.»

Ao. "Como que la Luna nos tiene amarrados.»

Ao. "Pero pesa más la Tierra y jalaría a la Luna."

M. "¿Cómo se llama esa fuerza?»

Ao. "Gravedad."

M. «¿stá en todo el espacio?»

Ao. «Pero a la Tierra no la puede sostener la gravedad, porque está en ella misma."

M. «Gabriela dice que no se cae porque al estar girando genera aire que no la deja irse.»

Ao. "Como usted dice hay gravedad en todo el espacio, y eso la sostiene como fuerza magnética.»

M. "¿Por qué magnética?"

As. "Porque es una fuerza que atrae»

Esta secuencia representa el nivel más alto de organización argumentativa del discurso durante esta clase ya que los niños confrontan alternativas explicativas y buscan argumentos en favor o en contra para tratar de entender ${ }^{7}$ por qué se sostiene la Tierra en el espacio.

Es significativo que esta dinámica sea iniciada con la pregunta de una alumna ("¿Por qué no nos caemos?») para la que no parece haber una respuesta preconstruida en el aula. Después de que el maestro devuelve la pregunta al grupo, la mayor parte de la interacción se establece entre los alumnos. Se muestra el valor constructivo de la relación entre pares cuando la interacción se centra en un problema significativo.

Las inquietudes de los niños y sus explicaciones rebasan la descripción argumentada, validada por contraejemplos y analogías, que venía solicitando el maestro. Su interés por el problema planteado hace que sus aportaciones adquieran una cierta autonomía de las pautas marcadas por el docente.

Los niños parecen estar tratando de entender por qué se mueven así los planetas y la razón por la que este movimiento difiere de otros que ellos conocen (¿por qué la Tierra se puede sostener en el espacio, si nuestra experiencia es que las cosas caen?, ¿ por qué los planetas siempre se están moviendo y además en una misma órbita, si eso no ocurre cotidianamente con los cuerpos en movimiento?). Recurren a diversos conocimientos, establecen analogías y comparaciones con otras experiencias para buscar una explicación que dé coherencia a sus ideas sobre las características del movimiento de los objetos y las causas que lo provocan. 
La primera explicación de que no nos caemos «porque hay una fuerza magnética que nos sostiene a nosotros» es cuestionada por la misma niña quien aclara que se refiere a quién sostiene a la Tierra. Parece, por las intervenciones posteriores, que ya conocen la idea de la gravedad como una fuerza que nos mantiene sobre la Tierra. Otra niña construye una explicación de por qué se sostiene la Tierra: «Porque al girar (la Tierra) genera aire que la sostiene.» De esta manera pone en juego lo que podríamos llamar una teoría en acción (Karmiloff e Inhelderder, 1975): el aire en movimiento puede sostener cuerpos pesados. Puede ser que la niña aluda a un modelo empírico, elaborado por analogía con fenómenos conocidos a partir de su experiencia práctica, que la lleva a explicar la permanencia de algunos objetos en el aire (papalotes o cometas, avioncitos de papel, etc.), por el efecto del movimiento del objeto con respecto al aire. Esta explicación no es comentada por otros alumnos, probablemente porque está muy distante de lo que ellos pueden concebir.

La construcción que hace esta niña (de 10 años) de un modelo analógico, por demás original, y su utilización para simular las relaciones de causa-efecto que pueden explicar por qué la Tierra se sostiene en el espacio, es semejante a la manera como en la ciencia se elaboran teorías y explicaciones a través de modelos metafóricos ${ }^{8}$.

Estos resultados relativizan algunas de las conclusiones que obtiene J. Solomon (1986) sobre las dificultades de los alumnos de secundaria para elaborar explicaciones utilizando relaciones causales y para construir analogías que vayan más allá de sustituir una palabra por otra.

Las intervenciones de los niños se van articulando en una confrontación argumentativa. La explicación de que «hay una fuerza magnética que nos jala para abajo", es cuestionada implícitamente por otro niño que plantea una explicación alternativa: "Como que la Luna nos tiene amarrados.» Este modelo suscita, inmediatamente, un argumento en contra: «Pero pesa más la Tierra y jalaría a la Luna." Para argumentar el niño utiliza conocimientos escolares y extraescolares como el tamaño y peso relativos de la Luna y la Tierra y el que un objeto más pesado no puede ser sostenido por uno menos pesado.

El maestro menciona la fuerza de gravedad y su ubicación en el espacio, pero un niño no acepta la explicación (implícita) de que la Tierra se sostiene por la gravedad, a pesar de que es propuesta por el maestro. Cuestionar la propuesta del maestro es algo poco frecuente en la escuela y tal vez puede explicarse porque en ese momento la necesidad de resolver el problema hace que la lógica del conocimiento adquiera una relevancia mayor que seguir la lógica de la interacción con el docente ${ }^{9}$.

Para argumentar en contra del comentario del maestro, el niño se apoya en su conocimiento escolar sobre la gravedad: «Pero a la Tierra no la puede sostener la gravedad porque está en ella misma» ${ }^{10}$. Después de la aclaración del niño, el maestro retoma el modelo sobre la Tierra en movimiento posiblemente para que se reflexione sobre él, pero de nuevo es ignorado por los demás niños. Otro alumno, tratando de sostener la idea de la gravedad, mencionada por el docente, explica: «Como usted (el maestro) dice, hay una gravedad en todo el espacio y eso la sostiene como fuerza magnética." El niño expresa la idea, de la gravedad en el espacio, parecida a un campo de fuerzas (como fuerza magnética) que sostiene a la Tierra. 
Esta es una confrontación de al menos tres modelos distintos para explicar por qué se mantiene la Tierra en el espacio:

- La Tierra se sostiene por el aire que genera al girar.

- La Tierra se sostiene por la atracción de otros astros («Como que la Luna la tiene amarrada»).

- A la Tierra la sostiene, como fuerza magnética, la gravedad que está en todo el espacio.

En esta confrontación los niños no se pronuncian frente al primer modelo, pero dan argumentos explícitos en contra de la segunda y la tercera explicación. Sin embargo en el discurso no queda descartada ninguna de las explicaciones.

Como dice Billig (1987) retomando a Protágoras, los más puros debates retóricos no pretenden llegar a un acuerdo, sino que fomentan la argumentación para que los alumnos se entrenen en encontrar las diversas facetas y contradicciones en un problema. El pensamiento humano se caracteriza por su variedad y la diversidad de argumentos siempre será más rica que la uniformidad de opiniones.

Este debate representa una elaboración sobre el conocimiento al plantear diferentes opciones explicativas que pueden operar como hipótesis y al dejar planteados los problemas y las contradicciones que los alumnos ven en estas explicaciones. Esta es, muchas veces, la forma como la ciencia se va aproximando a una nueva conceptualización sobre un fenómeno.

El debate es posible, en parte, porque el maestro no descarta ninguna explicación dando su opinión, sino que las mantiene como distintas opciones posibles. Asimismo, permite que se reflexione sobre ellas y que los propios alumnos las analicen al confrontarlas. De esta manera se incorporan las dudas y las opiniones de los alumnos como parte del proceso de construcción del conocimiento escolar y no se impone sin argumentos la posición del maestro o del Libro de Texto.

En la parte final de la clase el maestro vuelve a retomar el repaso preguntando, con un sentido otra vez finalista que en principio no propicia la reflexión, cuál es la función que tienen los planetas en el sistema. Los alumnos responden que rotar y trasladarse mientras que simultáneamente siguen analizando la dinámica del sistema a través de contraejemplos y de la alteración de variables que les permiten poner a prueba el modelo. Esto se puede ver en las preguntas que hacen los alumnos casi al final de la clase: «Maestro, si la Tierra girara muy rápido ¿se vería como una luz que se prende y se apaga?», «Si la Tierra se saliera de su órbita ¿a qué velocidad saldría?»

\section{CONCLUSIONES}

En esta clase se genera una dinámica de argumentaciones y elaboraciones explicativas, poco común en la escuela. Sin embargo, el hecho de que ésta no sea una clase preparada ni con un maestro especialmente capacitado o de los llamados innovadores, permite pensar que es posible que en otros casos también ocurran situaciones con estas características (Candela, 1989 y 1990). El análisis de las prácticas educativas reales (en este caso, de las 
prácticas de un buen maestro «tradicional») puede aportar información fundamental sobre las formas de ayuda, las intervenciones didácticas y las condiciones concretas de la interacción en el aula que propician o dificultan la construcción del conocimiento escolar. Esta información es de relevancia central para la elaboración de propuestas que pretendan mejorar la enseñanza en la escuela.

En este caso el control del docente sobre la dinámica de las interacciones está orientado alternativamente hacia un repaso de conocimientos escolares sobre el Sistema Solar y a demostrar la validez de este modelo. El maestro conduce la interacción con una estructura muy dirigida y en ocasiones hasta cerrada, pero utiliza recursos didácticos que llevan la intención de que los niños «entiendan" el contenido. Esta intencionalidad del maestro junto con la significatividad que el conocimiento así presentado va teniendo para los alumnos, contribuye a que ellos traten de entender las causas de los fenómenos analizados ${ }^{11}$.

La estructura de la interacción controlada y dirigida por el docente coexiste con una relación constructiva de los alumnos con el conocimiento. Se muestra que la estructura de la organización social del discurso al modo como lo concibe Mehan (1979), donde la forma condiciona el contenido, no es suficiente para explicar algunas dinámicas que se pueden desarrollar en el aula ni la relación de los alumnos con el conocimiento escolar, ya que Mehan no analiza la significatividad del contenido para los sujetos.

En este caso también se encuentra que la estrategia de memorización de datos y definiciones no se contrapone, sino que puede apoyar, a la relación constructiva con el conocimiento, si las expectativas del maestro no cierran la posibilidad de plantear explicaciones alternativas o nuevas dudas conceptuales.

Algunas características significativas del contexto que pueden explicar las condiciones que ayudan a que el maestro abra la posibilidad de una reelaboración del conocimiento son: que se trata de un maestro que tiene establecida una fluida dinámica de interacción con los alumnos, que tiene seguridad en el conocimiento del tema y que previamente se asegura de que los niños manejen el contenido escolar del mismo. En estas condiciones el maestro admite opiniones divergentes de los alumnos y negocia con ellos el conocimiento.

Los niños, siguiendo la dinámica planteada por el maestro, convierten una clase demostrativa en una clase argumentativa que termina problematizando el modelo del Sistema Solar.

Los alumnos desplazan la interacción del terreno de las preguntas ficticias del maestro, porque él ya sabe las respuestas (Stubbs, 1984), al terreno donde las soluciones no están dadas. El conocimiento se abre entonces a la búsqueda de explicaciones, de nuevas elaboraciones, poniendo en juego los conocimientos previamente adquiridos y tratando de dar coherencia a las ideas mas allá del discurso escolar. La interacción entre los alumnos y el maestro abre y cierra alternativamente el conocimiento de lo conocido a lo desconocido. Los recursos que el docente pone en juego para que acepten la descripción del Sistema Solar, los alumnos los utilizan para tratar de entender las causas de la dinámica del sistema. Se desarrolla así una construcción del significado (Tannen, 1989) del conocimiento social entre los alumnos y el maestro. La educación se convierte así en una producción co- 
lectiva donde el maestro tiene que negociar el conocimiento para mantener el control del proceso.

El proceso que siguen los niños estableciendo relaciones de causa-efecto, argumentando sus ideas, buscando explicaciones alternativas, tratando de comprender las leyes que explican la especificidad de un modelo de movimiento que contradice la experiencia cotidiana y, probablemente, dándose cuenta de que todo conocimiento es cuestionable y es necesario analizarlo y justificarlo, vincula sus intervenciones con el quehacer científico mucho más que seguir un esquema rígido denominado "método científico" y parece resultar formativo a pesar de no seguir ningún «modelo didáctico innovador» para mejorar la enseñanza de la ciencia.

Esta clase muestra que cuando el maestro abre la posibilidad de que los alumnos manifiesten sus opiniones y reflexionen sobre ellas, no importa si es con una estructura más o menos cerrada o si es antes o después de haber transmitido cierta información escolar, los alumnos, pueden rebasar las expectativas del docente y alterar la dinámica de la interacción al mismo tiempo que contribuir a nuevas construcciones del conocimiento. Esto es así siempre que el conocimiento resulte significativo para ellos.

Los niños en el aula pueden argumentar sus puntos de vista y elaborar explicaciones utilizando analogías complejas. Probablemente la diferencia entre los resultados de este trabajo y los de algunos estudios controlados sobre las concepciones y recursos explicativos de los alumnos (Solomon, 1986) puede ser explicada por el efecto del contexto social interactivo sobre las posibilidades de manifestar y enriquecer ciertas explicaciones.

De aquí que se considere la importancia central que tiene el contexto interactivo para analizar las posibilidades de la construcción del conocimiento de ciencias naturales y la necesidad de profundizar en trabajos que permitan comprender estos procesos sociales en toda su complejidad.

\section{Notas}

1 Los datos empíricos utilizados en este artículo se presentaron en: Candela, M.A. «La necesidad de entender, explicar y argumentar: los alumnos de primaria en la actividad experimental». Tesis de Maestría en Ciencias de la Educación. Departamento de Investigaciones Educativas. Centro de Investigación y Estudios Avanzados del Instituto Politécnico Nacional. México, 1989.

2 Para Piaget, iniciador de este tipo de estudios, las estructuras mentales se forman en la interacción con el mundo físico y sólo después de que estas estructuras existen, el individuo puede comprender o asimilar la información que proviene de la interacción social. Esta postura ha sido cuestionada por enfatizar demasiado la actividad autoestructurante y conducir a una visión individualista de los procesos educativos escolares (Bruner, 1984, Coll, 1986).

${ }_{3}^{3}$ Para C. Coll (1978) el objetivo de la enseñanza de las ciencias naturales es el contribuir a la formación de una actitud científica, entendida ésta como la formulación de hipótesis y su verificación posterior a través de las experiencias adecuadas.

4 El trabajo de campo se realizó en 1985, dentro de un estudio cualitativo para analizar la enseñanza de las ciencias naturales en la escuela primaria bajo mi coordinación y con el apoyo, como auxiliares de investigación, de Ana Isabel León y Norma Venegas, en el Departamento de Investigaciones Educativas.

5 En el libro de texto de Ciencias Naturales la lección sobre el Sistema Solar en $5 .^{\circ}$ grado se inicia con la historia de una niña (Juanita) que estaba con su perrito (Canica) y se queda dormida. Sueña con que va en una nave espacial visitando los planetas y observando sus características.

${ }_{6}^{6} \mathrm{La}$ explicación que solamente describe un fenómeno y que tiene rasgos de finalismo, es una de las formas más elementales de explicación (Piaget, 1972). Desde esta perspectiva po- 
driamos decir que la complejidad de las explicaciones dadas hasta este momento por los alumnos parece-ser mayor que la de las explicaciones del maestro.

7 Entender un fenómeno para Johnson-Laird (1983) es tener un modelo dinámico de él. Un modelo que tenga algunos elementos simulados.

${ }^{8}$ Hesse (1966) hlama redescripción metafórica a la explicación científica. Joan Solomon (1986) plantea que explicar por comparación usando un modelo o analogía es un método científico particular. Harré (1983) dice que la metáfora que sugiere relaciones de causa-efecto es el tipo más complejo de analogía.

En el trabajo de Rockwell y Gálvez (1982) se desarrollaron las categorías de lógica de la interacción y lógica del contenido como las dos lógicas que tiene que seguir simultáneamente un alumno en la dinámica de la interacción en un aula. El análisis de la relación entre estas dos lógicas ha sido utilizado en algunos de los trabajos realizados en el DIE (Paradise, 1979, Edwards, 1988, Candela, 1989 y 1990) para entender las características de algunas situaciones de comunicación que se dan en el aula.

${ }^{10}$ En la escuela se explica a los niños que nos sostenemos sobre la superficie de la Tierra porque somos atraídos hacia su centro por la fuerza de gravedad pero generalmente no se abor$\mathrm{da}$ la concepción de la gravedad como la fuerza que existe entre dos masas cualquiera, aunque ésta es planteada en el Libro de Texto.

${ }_{11}$ Las intervenciones didácticas del maestro que propician la participación argumentativa de los alumnos son la siguientes:

- Devolver las preguntas al grupo.

- Permitir la producción de explicaciones alternativas sobre los fenómenos observados.

- Propiciar que se ponga en duda el saber recibido como evidente.

- Propiciar que se escuchen y analicen otras opciones explicativas.

- Promover que se busque la coherencia entre el conocimiento que se estudia, lo que observan y hacen los niños con los saberes previos que tienen (tanto escolares como extraescolares).

- Pedir que se argumenten las explicaciones para convencer sobre su pertinencia o validez.

- Aceptar el error como parte del proceso de construcción.

\section{Referencias}

BARNES, D. (1971). *Language and learning in the classroom*. Journal of Curriculum Studies, 3, I. 101.

BilLIG, M. (1987). Arguing and Thinking: a Rethorical Approach to Social Psychology. Cambridge: Cambridge University Press.

Bruner, J. (1984). Acción, Pensamiento y Lenguaje. Comp. J. L. Linaza. Madrid: Alianza-Psicología.

CANDELA, A. (1989). *La necesidad de entender, explicar y argumentar: los alumnos de primaria en la actividad experimental.. Tesis de Maestría en Ciencias. México: Departamento de Investigaciones Educativas. Centro de Investigación y Estudios Avanzados. Instituto Politécnico Nacional.

CANDELA, A. (1990). «Investigación etnográfica en el aula: el razonamiento de los alumnos en una clase de ciencias naturales en la escuela primaria." Investigación en la Escuela, 11.

CiCOUREL, A (1974). «Some basic theoretical issues in the assessment of the child's performance in testing and classroom settings». En Language Use and School Performance.

ColL, C. (1986). «Acción, interacción y construcción del conocimiento en situación escolar». Revista de Educación, 279, 9-25.

CoLL, C. (1978). La conducta experimental en el niño. Barcelona: CEAC

DRIVER, R. (1981). «Pupils' alternative frameworks in science*. Eur. J. Scie. Ed. 3, 93-101.

DrIVER, R.; GUESNE, E. y ThIBERgIEN, A. (1985). Children's Ideas in Science. London: Open Univ. Press. Trad. al castellano como Ideas Científicas en la Infancia y la Adolescencia. España: MEC-Morata. 1989.

EDWARDS, D. y MERCER, N. (1987). Common Knowledge: the Development of Understanding in the Classroom. London: Routldge. Trad. al castellano como El Conocimiento Compartido: el Desarrollo de la Comprensión en el Aula. Temas de Educ. Barcelona: PaidósMEC. 1988.

EDWARDS, D (1990a). «Classroomm discourse and classroom knowledge». En C. Rogers y P. Kutnick (Eds.), The Social Psychology of the Primary School. London: Routledge.

EDwards, D. (1990b). *El papel del profesor en la construcción social del conocimiento*. Investigación en la Escuela, 10, 33-49.

EDWARDS, D. (en prensa). *Concepts, memory, and the organization of pedagogic discourse: a case study m. International Journal of Educational Research. 
Gilbert, G. N. y Mulkay, M. (1984). Opening Pandora's Box: A Sociological Analysis of Scientists' Discourse. Cambridge: Cambridge University Press.

HERNÁNDEZ, J. (1989). «La enseñanza de las ciencias naturales: entre una redescripción de la experiencia cotidiana y una resignificación del conocimiento escolar». Tesis de Maestría en Ciencias. Departamento de Investigaciones Educativas. Centro de Investigaciones y Estudios Avanzados. Instituto Politécnico Nacional. México.

HARRE, R. (1983). "History and philosophy of science in the pedagogical process*. En: Home (Ed.), Science Under Scrutiny, 139-158. Dordrecht: Reidel.

Hesse, M. (1966). Models and Analogies in Science. University of Nôtre Dame Press.

JoHNSON-LAIRD, P. (1983). Mental Models. Londres: Cambridge University Press.

LEITH, D. y MYERSON, G. (1989). The Power of Address: Explorations in Rhetoric. London and New York: Routledge.

LONGINO, H. (1990). Science as a Social Knowledge. Values and Objetivity in Scientif Inquiry. Princeton, New Jersey: Princeton University Press.

Mehan, H. (1979). Leaming Lessons: Social Organization in the Classroom. London: Harvard University Press.

KARMILOFF, A. e INHELDER, B. (1975). «Si quieres avanzar hazte con una teoria». Cognition International Joumal of Cognitive Psychology. 3 (3).

PHILlips, D. C. (1985). "On what scientist know, and how they know it». En Elliot Eisner (Ed.) Learning and Teaching the Ways of Knowing. 84 year book of the National Society for the Study of Education, 37-60.

PiAgET, J. (1972). El Juicio y el Razonamiento en el Niño. Madrid: La Lectura.

PIAGET, J. (1973). La Representación del Mundo en el Niño. Madrid: Morata.

ROCKWELL, E y GALVEZ, G. (1982). *Formas de transmisión del conocimiento científico, un análisis cualitativo». Educación: 42, 97-139. México: Consejo Nacional Técnico de la Educación, Secretaria de Educación Pública.

SOLOMON, J. (1986). *Children's explanations». Oxford Review of Education, 12 (1), 41-51. STUBBS, M. (1984). Lenguaje y escuela: análisis sociolingüistico de la enseñanza, Dialogos en Educación 19, Madrid. Cincel-Kapelusz.

TANNEM, D. (1989). Talking Voices. Cambridge: Cambridge University Press.

VIENNOT, L. (1979). «Spontaneous reasoning in elementary dynamics». Eur. J. Scie. Ed. 1 (2), 205-221.

\section{Extended summary}

Psychological research dominates the teaching of Natural Sciences and contributes with important data on the different conceptions children have about scientific topics, but it gives little information about how they construct their knowledge in the school context. It is important to study both the individual cognitive conditions and the interactive context in order to understand the processes which help to produce educational changes.

The interactive patterns which help us to understand how knowledge is socially constructed in an elementary classroom are analyzed in this paper. It presents an ethnographic description of the interaction between the teacher and the students in a primary school lesson on the Solar System, taught by a "good traditional" teacher. The assumption is that research on teaching practices supplies knowledge about the conditions which favor learning in the school context. This knowledge has not been sufficiently considered, particulary in science teaching.

The analysis focuses on the students' interventions in which they formulate alternative explanations and argue their ideas. These are relevant forms of participation in scientific training. The study of argumentation has become increasingly important in the new sociology of scientific knowledge (Gilbert y Mulkay, 1984). Furthermore, the argumentative organization of discourse is an important base for conceptual reasoning (Billig, 1987, Leith and Myerson, 1989). 
The study shows that even when the teacher establishes interaction rules with a closed structure, his interest in demonstrating the validity of the Solar System model leads the students to a reflexive relation with knowledge. The dynamics of interaction depend not only on the form and structure of the interaction patterns, but also on the significance of the contents for the interacting individuals.

Some of the teacher's didactic interventions promote a constructive relation with knowledge, including meaningful answers and alternative explanations given by the students, confrontation between different points of view, use of arguments, counterexamples and analogies to validate school knowledge.

The teacher in this class promotes open participation. Some conditions which seem relevant in this process are: the flow in the interaction dynamics established by the children, the teacher's knowledge of the subject, and his awareness that the children have already acquired certain basic school information about of the Solar System.

Within this context, the students, using the didactic resources the teacher gives them, modify the logic of the discourse. They turn the demonstration of the planetary model into a problematization of the model. Students are shown to be active individuals who alter the dynamics of interaction and contribute significantly to the construction of classroom know ledge.

Pseudo-questions asked by the teacher elicit open questions from the children. They try to find explanations for the dynamics of the Solar System (How come the planets don't crash? What would happen if the planets stopped moving? Why don't we fall down? Who holds up the Earth?) The children produce tentative answers, analogies and more complex models (The Earth doesn't fall because in turning it generates the air that sustains it). They modify the variables in the model (If the Earth turned faster, would it look like a light that turns on and off?). They analyze counterexamples (The Earth cannot show only one face to the Sun, "It would be cold on one side and hot on the other", "There wouldn't be day and night or seasons»). They elaborate and argue alternative explanations ("It seems like themoon is holding us», "But the moon is heavier and it would pull the Earth"), and they correlate school and out of school information which is related to the problems they try to solve.

The meaning of the interactive context for the children and the relevance of the teacher's didactic interventions allow a negotiated construction of scientific knowledge. This kind of argumentation of real problems surely promotes the students' scientific training more than any didactic model derived from a context outside school. 